\title{
An unusual combination of large Eustachian valve in a young patient with Friedreich's ataxia cardiomyopathy
}

\author{
Stylianos A. Karvounaris ${ }^{1}$, Georgios S. Papaetis ${ }^{2,3} \odot$, Petros P. Mavrommatis ${ }^{4}$ \\ ${ }^{1}$ Department of Cardiology, Evangelismos Hospital, Paphos, Cyprus \\ ${ }^{2}$ Internal Medicine and Diabetes Clinic, Paphos, Cyprus \\ ${ }^{3} \mathrm{CDA}$ College, Paphos, Cyprus \\ ${ }^{4}$ Cardiac Care Center, Paphos, Cyprus
}

A 20-year-old male with Friedreich ataxia (FA) presented with dyspnoea and palpitations over the prior 2 days. Atrial fibrillation with rapid ventricular response and inverted $\mathrm{T}$ waves in leads III, aVF, V5, V6 were found on the electrocardiogram. The transthoracic echocardiogram, with the patient being in atrial fibrillation, showed concentric hypertrophy and left ventricular ejection fraction of approximately $50 \%$. Surprisingly, an incomplete membrane into the right atrium was noted (Fig. 1A, B). The cardiac rhythm was restored to sinus rhythm after 24 hours. Further study with transesophageal and contrast echo clearly demonstrated the presence of an incomplete membrane dividing the right atrium into two chambers (Fig. 1C, D). Color and pulsed Doppler showed a turbulent eccentric flow across the two portions of the right atrium, without a significant gradient, expressing a large Eustachian valve or an incomplete cor triatriatum dextrum. Moreover, the presence of a patent foramen ovale noted and the left atrial appendage was free of thrombi. Agitated contrast saline infusion enhanced clarification of the anatomy. The turbulent and eccentric flow was crossing the 'hole' of the membrane and entering the proximal right atrium cavity, swirling in it, before moving through the distal right atrium cavity to the tricuspid valve relatively unconstructively (Fig. 1E, F). Cardiac magnetic resonance imaging was performed and documented the presence of large Eustachian valve in the presence of cardiomyopathy (FA-CM). The patient is under close echocardiographic follow-up. According to available research this is the first published description of a large Eustachian valve in a patient with FA-CM.

Conflict of interest: None declared

Address for correspondence: Georgios S. Papaetis, MD, PhD, Internal Medicine and Diabetes Clinic, Eleftherios Venizelos Avenue 62, Paphos, Cyprus, tel: 0035799273573, fax: 0035726220451, e-mail: gpapaetis@yahoo.gr 


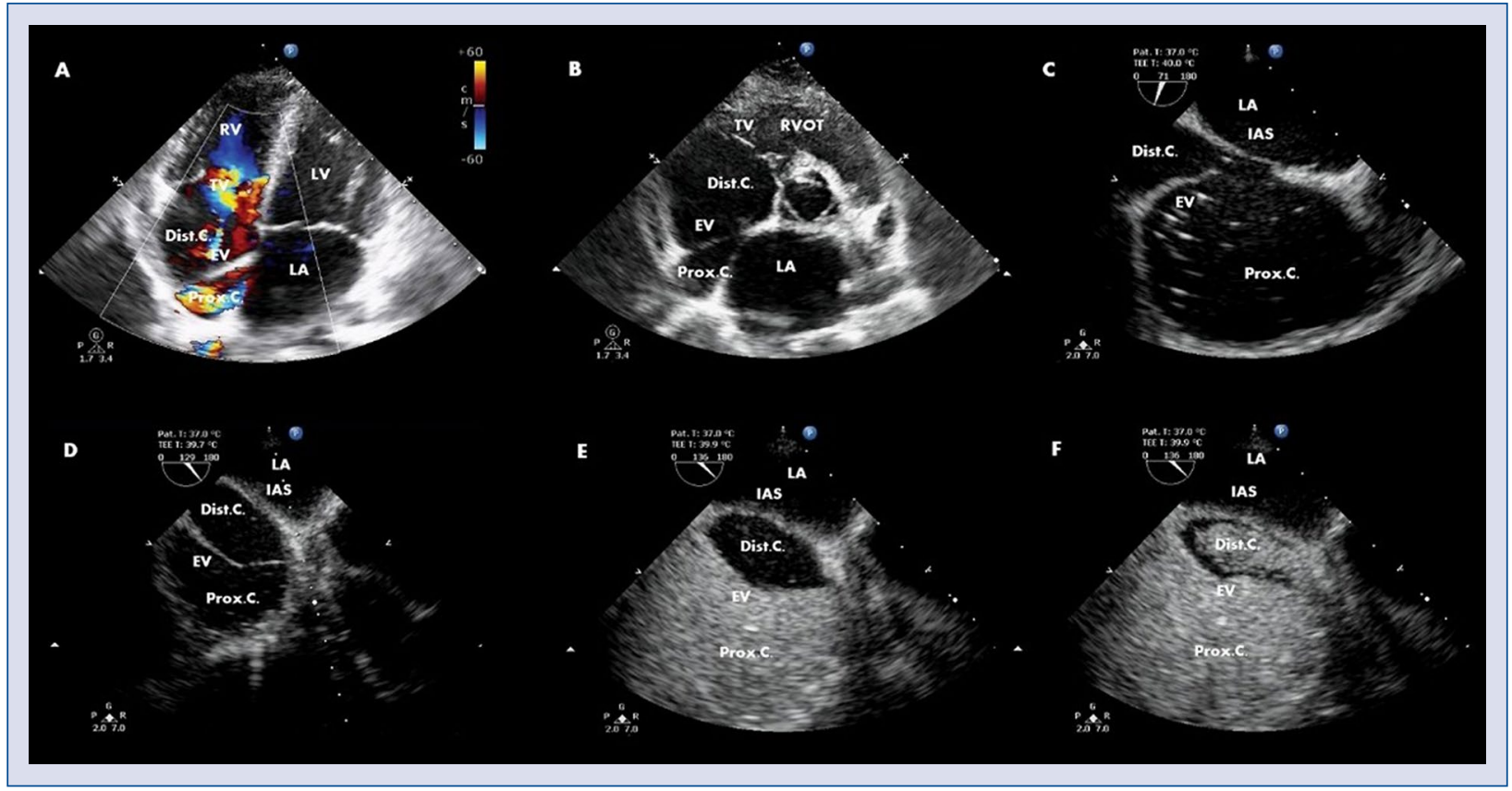

Figure 1. A. A large Eustachian valve divides the right atrium in two cavities (transthoracic echocardiogram [TTE] 4C); B. TTE SAX; C. Transesophageal echocardiogram (TEE) $70^{\circ}$; D. TEE $130^{\circ}$. Agitated contrast saline infusion initially fills the proximal right atrium (RA) cavity only; E. Later the contrast enters the distal RA cavity; F. Echo contrast free space between the two RA cavities represents the Eustachian valve (EV); LV — left ventricle; RV — right ventricle; LA — left atrium; Prox.C — proximal right atrium cavity; Dist.C — distal right atrium cavity; IAS — interatrium septum; TV — tricuspid valve. 